University of Wollongong

Research Online

Australian Institute for Innovative Materials -

Papers

Australian Institute for Innovative Materials

$1-1-2019$

\title{
Bio-Inspired Stretchable and Contractible Tough Fiber by the Hybridization of GO/MWNT/Polyurethane
}

\author{
Hyunsoo Kim \\ Hanyang University \\ Yongwoo Jang \\ Hanyang University \\ Dong Yeop Lee \\ Hanyang University \\ Ji Hwan Moon \\ Hanyang University \\ Jung Gi Choi \\ Hanyang University
}

See next page for additional authors

Follow this and additional works at: https://ro.uow.edu.au/aiimpapers

Part of the Engineering Commons, and the Physical Sciences and Mathematics Commons

Research Online is the open access institutional repository for the University of Wollongong. For further information contact the UOW Library: research-pubs@uow.edu.au 


\title{
Bio-Inspired Stretchable and Contractible Tough Fiber by the Hybridization of GO/ MWNT/Polyurethane
}

\author{
Abstract \\ Spider silks represent stretchable and contractible fibers with high toughness. Those tough fibers with \\ stretchability and contractibility are attractive as energy absorption materials, and they are needed for \\ wearable applications, artificial muscles, and soft robotics. Although carbon-based materials and \\ poly(vinyl alcohol) (PVA) composite fibers exhibit high toughness, they are still limited in low extensibility \\ and an inability to operate in the wet-state condition. Herein, we report stretchable and contractible fiber \\ with toughness that is inspired by the structure of spider silk. The bioinspired tough fiber provides $495 \mathrm{~J} / \mathrm{g}$ \\ of gravimetric toughness, which exceeds $165 \mathrm{~J} / \mathrm{g}$ of spider silk. Besides, the tough fiber was reversibly \\ stretched to $-80 \%$ strain without damage. This toughness and stretchability are realized by hybridization \\ of aligned graphene oxide/multiwalled carbon nanotubes in a polyurethane matrix as elastic amorphous \\ regions and $\beta$-sheet segments of spider silk. Interestingly, the bioinspired tough fiber contracted up to \\ $60 \%$ in response to water and humidity similar to supercontraction of the spider silk. It exhibited $610 \mathrm{~kJ} /$ \\ $\mathrm{m} 3$ of contractile energy density, which is higher than previously reported moisture driven actuators. \\ Therefore, this stretchable and contractible tough fiber could be utilized as an artificial muscle in soft \\ robotics and wearable devices.

\section{Disciplines} \\ Engineering | Physical Sciences and Mathematics

\section{Publication Details} \\ Kim, H., Jang, Y., Lee, D. Yeop., Moon, J. Hwan., Choi, J. Gi., Spinks, G. M., Gambhir, S., Officer, D. L., \\ Wallace, G. G. \& Kim, S. Jeong. (2019). Bio-Inspired Stretchable and Contractible Tough Fiber by the \\ Hybridization of GO/MWNT/Polyurethane. ACS Applied Materials and Interfaces, 11 31162-31168.

\section{Authors} \\ Hyunsoo Kim, Yongwoo Jang, Dong Yeop Lee, Ji Hwan Moon, Jung Gi Choi, Geoffrey M. Spinks, Sanjeev \\ Gambhir, David L. Officer, Gordon G. Wallace, and Seon Jeong Kim
}


Bio-Inspired Stretchable and Contractible Tough Fiber by the Hybridization of GO/MWNT/Polyurethane

Hyunsoo Kim, ${ }^{1}$ Yongwoo Jang, ${ }^{1}$ Dong Yeop Lee, ${ }^{1}$ Ji Hwan Moon, ${ }^{1}$ Jung Gi Choi, ${ }^{1}$

Geoffrey. M. Spinks, ${ }^{2}$ Sanjeev Gambhir, ${ }^{2}$ David L. Officer, ${ }^{2}$ Gordon G. Wallace, ${ }^{2}$ and Seon Jeong $\mathrm{Kim}^{1 *}$

${ }^{1}$ Center for Self-powered Actuation, Department of Biomedical Engineering, Hanyang University, Seoul 04763, Korea

${ }^{2}$ Intelligent Polymer Research Institute, ARC Centre of Excellence for Electromaterials Science, AIIM Facility, Innovation Campus, University of Wollongong, North Wollongong, NSW 2522, Australia

*To whom correspondence should be addressed, E-mail: sjk@ hanyang.ac.kr 


\begin{abstract}
Spider silks represent stretchable and contractible fibers with high toughness. Those tough fibers with stretchability and contractibility are attractive as energy absorption materials, and are needed for wearable applications, artificial muscles, and soft robotics. Although carbonbased materials and poly (vinyl alcohol) (PVA) composite fibers exhibit high toughness, it is still limited in low extensibility and inability to operate in the wet-state condition. Herein, we report stretchable and contractible fiber with toughness that is inspired by the structure of spider silk. The bio-inspired tough fiber provides $495 \mathrm{~J} / \mathrm{g}$ of gravimetric toughness, which exceeds 165 $\mathrm{J} / \mathrm{g}$ of spider silk. Besides, the tough fiber was reversibly stretched to $\sim 80 \%$ strain without damage. This toughness and stretchability are realized by hybridization of aligned graphene oxide/multi-walled carbon nanotubes in a polyurethane matrix as elastic amorphous regions and $\beta$-sheet segments of spider silk. Interestingly, the bio-inspired tough fiber contracted up to $60 \%$ in response to water and humidity similar to supercontraction of the spider silk. It exhibited $610 \mathrm{~kJ} / \mathrm{m}^{3}$ of contractile energy density, which is higher than previously reported moisture driven actuators. Therefore, this stretchable and contractible tough fiber suggests to be utilized as an artificial muscle in soft robotics and wearable devices.
\end{abstract}

Keywords: Toughness, stretchability, contraction, carbon nanotube, graphene oxide, polyurethane, fiber 


\section{Introduction}

Tough materials are greatly valued in load-bearing applications because of their ability to absorb mechanical energy before fracture. Recent studies have focused on enhancing the toughness of fibers or yarns, ${ }^{1-3}$ hydrogels, ${ }^{4-8}$ and composites ${ }^{9,10}$ and applying these materials in bulletproof vests, wearable devices, and artificial muscle. A particularly successful strategy for enhancing fiber toughness has been the use of nano-carbon materials as toughening agents when added to various binders. In these and other studies the toughness of fibers has been approximated by the work of extension that is obtained from the area beneath the stress-strain curve. Dalton et al., for example, demonstrated tough fibers with a work of extension of 570 $\mathrm{J} / \mathrm{g}$ and that were composed of carbon nanotubes in a poly (vinyl alcohol) (PVA) matrix. ${ }^{1}$ Also, our group showed the highest toughness in a fiber $(970 \mathrm{~J} / \mathrm{g})$ by the synergistic effect resulting from the combination of reduced graphene oxide flakes and single-walled carbon nanotubes in a PVA matrix. ${ }^{11}$ The practical usefulness of these PVA based fibers was restricted, however, by their dramatic loss of strength when exposed to water which is understood as the PVA is water soluble. Secondly, our previous tough fibers could only sustain strains to $\sim 20 \%$ before yielding and so restricting applications where high stretchability is desired, such as stretch fabrics.

Here, we demonstrate a functional tough fiber, which is water-insoluble, stretchable, and shows high contractions on exposure to moisture. The fibers were inspired by both the microstructure and properties of spider silk. Fibers prepared from spider silk are well-known for their high toughness of $165 \mathrm{~J} / \mathrm{g}^{12}$ that has been linked to the energy absorbing behavior of the silk microstructure during deformation. More interestingly, the spider silk has an ability to absorb water which can also cause contractions of $>50 \%$ of its original length. This behavior is sometimes called supercontraction ${ }^{13}$ and is thought to be an important biological process that enables the spider web to recover damage induced by wind or impact simply by the action of morning dew forming on the web. At the microstructural level, supercontraction occurs by the 
entropy-driven recoiling of molecular chains that had been first trapped in extended chain conformations by exposure to external loads in excess of the dry silk yield stress. ${ }^{14,15}$ Absorbed water plasticizes the silk to open up molecular free volume that facilitates the retraction of chains to their high entropy, random coil conformations with concomitant macroscopic strain recovery.

To mimic the silk behavior, we chose a hydrophilic polyurethane as the base material and added nano-carbons (graphene oxide (GO) and multi-walled carbon nanotubes (MWNT)) as toughening agents. This bio-inspired tough fiber was produced by a wet-spinning technique whereby the fiber-forming components were first dissolved and suspended in a spinning dope solution that was then injected into a coagulation bath of water. Through optimization of the composition and fabrication processes, the bio-inspired tough fibers were produced that demonstrate a toughness in the dry state of $494 \mathrm{~J} / \mathrm{g}$ and are highly stretchable with the ability to sustain $\sim 80 \%$ fully reversible strain over multiple stretch-relax cycles. Even in the wet state the fibers retain a high toughness of $456 \mathrm{~J} / \mathrm{g}$ and a strain at break of over $1200 \%$. The dry fibers contract in length by $\sim 60 \%$ on exposure to air of high humidity. Previously, the moisture driven artificial muscles were reported using by poly(diallyldimethylammonium chloride) infiltrated carbon nanotube yarn, ${ }^{16}$ and pentaerythritol ethoxylate and polypyrrole composite film. ${ }^{17}$ The toughness $(494 \mathrm{~J} / \mathrm{g})$ and the maximum contractile energy density $\left(610 \mathrm{~kJ} / \mathrm{m}^{3}\right)$ by a water-driven length contraction against a constant load are both higher than spider silk ${ }^{12}$ and previous moisture driven artificial muscles, and skeletal muscle. ${ }^{16-19}$

\section{Results and discussion}

The modified wet spinning process for preparing tough fibers is shown in Figure 1a. A spinning dope solution was composed by $1 \mathrm{wt} \%$ of nano-carbons (a mixture of MWNT and GO) and $8 \mathrm{wt} \%$ of the PU in the ethanol:water $(95: 5)$ solvent. Deionised water was used in the coagulation bath. The hybrid solution was prepared by a previously reported method which 
induces hydrogen bonding between the edge of GO and MWNT using sodium dodecyl benzene sulfonate (SDBS) ${ }^{11,20}$. The spinning dope was injected into the bottom of a water-filled glass tube and exploited the lower density of the ethanol-based hybrid solution compared with the water coagulation bath to vertically draw the fiber to the surface (Movie S1). The gel-state tough fibers could be removed from the coagulation bath and dried with mechanical stretch. In this manner, we obtained mechanically robust bio-inspired fibers of GO/MWNT imbedded in a PU matrix (Figure 1b). The schematic images of Figure 1b show the hydrogen bonded GO/MWNTs to be well-aligned in the PU matrix. This alignment has been shown to be induced by the high aspect ratio of GO/MWNT when the solution is extruded through the needle during the wet spinning process. ${ }^{11}$ The aligned and inter-connected network of GO/MWNT contribute to mechanical stiffness, strength and toughness and assume a similar role of $\beta$-sheets in spider silk. The elastomeric PU matrix contributes to the high strain extensibility in the fiber and acts like the amorphous phase in spider silk. The bio-inspired tough fibers were robust and could be knotted (Figure 1c) and twisted with multi-strands to form yarns (Figure 1d).

The mechanical properties for the bio-inspired tough fiber were measured by a tensile testing machine (UTM). All tensile testing was performed at a loading rate $1 \mathrm{~mm} / \mathrm{min}$. Figure 2a shows the maximum tensile stress and strain for dried tough fibers prepared using different ratios of GO and MWNT. Previous studies have shown that the fiber toughening is very sensitive to the ratio of GO and MWNT. ${ }^{11,21}$ Also, it has been reported that the mechanical properties depending on the ratio of the proteins even the spider silk. ${ }^{22}$ Figure S1 shows the thermal degradation behavior of the neat PU and GO/MWNT/PU fiber as determined by thermogravimetric analysis (TGA). As a result of TGA, GO/MWNT is contained around $20 \mathrm{wt} \%$ in the PU matrix because the weight of PU and GO/MWNT/PU fiber decreased to $2.4 \%$ and $22.3 \%$ by $800^{\circ} \mathrm{C}$, respectively. The highest strength observed was $152 \pm 24 \mathrm{MPa}$ and occurred at 3:7 weight ratio of GO:MWNT. The highest strain at break of $672 \pm 51 \%$ was produced by the fiber with a 5:5 (GO:MWNT) ratio. The stress-strain curves for fibers prepared 
with varying GO:MWNT ratios are shown in Figure S2. We estimate the volumetric toughness as the work of extension given by the area under the stress-strain curve. The tough fiber having a 5:5 (GO:MWNT) ratio showed the highest toughness, with an average $543 \mathrm{MJ} / \mathrm{m}^{3}$. Also, a gravimetric toughness, which is the equivalent mass-normalized toughness, was $494 \mathrm{~J} / \mathrm{g}$ (Figure 2b). This value was considerably higher than that of spider silk (165 J/g), ${ }^{12} \mathrm{CNT} / \mathrm{PU}$ (36 $\left.\mathrm{MJ} / \mathrm{m}^{3}\right),{ }^{23} \mathrm{MWNT} /$ polypropylene $(108 \mathrm{~J} / \mathrm{g}),{ }^{24}$ and Kevlar $(78 \mathrm{~J} / \mathrm{g}){ }^{25}$

The mechanical synergistic effect of GO and MWNT is highlighted by the results shown in Figure 2c. The strength and elongation at break of the tough fiber is superior to GO/PU, and MWNT/PU fiber, so that the toughness of the bio-inspired tough fiber is at least three times higher than GO/PU (32 J/g), and MWNT/PU $(130 \mathrm{~J} / \mathrm{g})$ fiber. The reinforcement mechanism generated by GO or CNT materials are well-known and variously reported. ${ }^{11,26,27}$ Seyedin et al. reported the reinforcement of thermoplastic PU composite fiber using GO. ${ }^{28}$ Wang et al. demonstrated a synergistic benefit when reinforcing fiber with a combination of $\mathrm{GO}$ and $\mathrm{CNT} .{ }^{27} \mathrm{Nah}$ et al. proved that an important factor for reinforcement of polymers by CNTs is the high aspect ratio of the CNT, causing physical entanglement and effective load transfer, rather than strong interfacial adhesion. ${ }^{29}$ The aligned network of GO and CNT effectively deflects cracks and prevents premature failure. ${ }^{30,31}$ Therefore, in the case of our tough fibers, the overall mechanical properties such as a modulus, strength, strain, and toughness were increased from $104 \mathrm{MPa}, 52 \mathrm{MPa}, 218 \%$, and $66 \mathrm{~J} / \mathrm{g}$ obtained in neat PU fiber (Figure S3) to $204 \mathrm{MPa}, 114 \mathrm{MPa}, 672 \%$, and $494 \mathrm{~J} / \mathrm{g}$, respectively, for tough fibers produced with GO and MWNT. The bio-inspired tough fibers show a doubling in the mechanical strength and tensile modulus when compared to the neat PU. In addition, the enhanced resistance to damage is particularly demonstrated by the trebling in the elongation at break. Consequently, the combined increase in strength and extensibility improves to produce 7.48 times in toughness of our optimized tough fiber in comparison to the unmodified PU fiber. 
Additionally, the stretching of the tough fibers after wet spinning and during the drying phase was found to have a powerful effect on the fiber mechanical properties. As shown in Figure S4, the stress-strain curves of the dry and stretched tough fibers are dramatically changed by the stretch ratios in the range of $0-500 \%$. Increasing the stretch ratio caused the fiber strength to increase while the elongation at break point decreased. These contrasting effects are likely due to the degree of alignment of the carbon nanoparticles. Jin et al. reported that mechanical stretching enhanced the alignment of MWNT in polymer-based composites. ${ }^{32}$ Our fibers stretched by $250 \%$ showed the highest toughness as indicated by the largest area under the stress-strain curve.

The alignment of the GO/MWNT in the tough fibers was investigated to confirm the role of nano-carbon alignment in enhancing the mechanical properties. Comparing the polarised Raman intensity ratio of the $\mathrm{G}$ band for polarization parallel and perpendicular of the fiber axis provided a measure of nano-carbon alignment because the intensity of $\mathrm{G}$ band is very sensitive to the orientation of CNTs. ${ }^{33} \mathrm{We}$ found that the Raman intensity ratio of $\mathrm{G}$ band increased from 1.46 to 2.04 after stretched the fiber by $250 \%$, indicating that GO/MWNT provided the increasing alignment from stretching (Figure $3 a, b$ ). The increasing alignment by stretching is attributed to a decrease in fiber diameter of $-44.3 \%$ (Figure S5). PU-free fibers were prepared for further evaluation by soaking the as-drawn fiber in ethanol solvent overnight and drying. Cross-sectional SEM images of the $250 \%$ stretched fiber and after removal of the PU matrix also illustrates a well-aligned network of GO/MWNT particles with alignment in the fiber direction (Figure 3c).

A cyclic response of the optimally prepared tough fibers to high strain extension is shown in Figure 4. Full strain reversibility was observed in stretch-relax cycles up to $80 \%$ strain (Figure $4 \mathrm{a}$ ). Yielding is observed at $\sim 80 \%$ strain from the stress-strain curve for the fiber (Figure 2c) so that some irrecoverable strain occurs beyond this point. Photographs of the $80 \%$ stretched and relaxed fibers with full reversibility are shown in Figure S6. Furthermore, there is no 
dramatic change in the deformation after 100 cycles of stretching and releasing (Figure $4 \mathrm{~b}$ ). The loading and unloading of the fibers generates a significant hysteresis. The hysteresis loops are usually attributed to detachment of rubber molecules between fillers and matrix and is called the Mullins effect that is a well-known characteristic of reinforced rubber. ${ }^{34}$ The detachment and re-attachment of the macromolecules from the filler surface is also known to contribute to high toughness. The reversibly stretchable tough fiber is attractive for wearable applications and superior to previous reported tough fibers from PVA or other materials such as 10,12 pentacosadiyn-1-ol (PCDA) that could not demonstrate stretchability. ${ }^{1,10,11}$ Also, unlike the previously described PVA based tough fibers, our bio-inspired tough fiber was found to be resilient to exposure to water. The mechanical properties of the wet-state fiber is shown in Figure 5. Although the mechanical strength decreased from 114 to $72 \mathrm{MPa}$, the elongation at break of the tough fiber increased from $673 \%$ to $1236 \%$ and the toughness only decreased by 8.5\% compared with dry-state fiber.

The water responsiveness of the tough fibers is another intriguing property of these materials. Figure 5 shows the change in length of the tough fiber when exposed to cycles of low and high humidity. When the relative humidity $(\mathrm{RH})$ around the tough fiber was increased steadily from $10 \sim 80 \%$ under $0.1 \mathrm{MPa}$ tensile load, a large contraction of the tough fiber occurred when the RH exceeded $20 \%$. The observed contraction was $59 \%$ of the initial dry length at RH $40 \%$ (Figure 6a). The contraction by humidity is shown in Movie S2. After this initial contraction, the tough fiber elongated $\sim 16 \%$ (normalized to the contracted length) when dried at the same constant load. Subsequent wet and dry cycles reversibly contract by water and expand by desiccation (Figure S7). Alternatively, by applying a higher mechanical stress to the dry fiber to restore its initial dry length, the large super-contraction is repeatable for humidifying cycles (Figure 6b). The work capacity obtained during super-contraction increased from 0.03 to $0.48 \mathrm{~J} / \mathrm{g}$, whereas the contraction strain decreased from 58 to $22 \%$ as the load applied increased from 0.1 to $2.5 \mathrm{MPa}$ (Figure 6c). When converted to volumetric energy 
density, the contractile work capacity of the tough fiber peaked at $610 \mathrm{~kJ} / \mathrm{m}^{3}$. These behaviors are strikingly similar to that of spider silk where super-contraction occurs on first contact with water and subsequent drying and wetting produce smaller, reversible length changes. ${ }^{18}$ As summarized in Figure 6d, the contractile work capacity of our fibers is higher than natural spider silk, and reported artificial muscle, actuators, and mammalian skeletal muscle. ${ }^{16-19,35}$ The reason for contraction is attributed to the rearrangement of the stretched polymer chains by water absorption because PU is ether-based hydrophilic polyurethane so that the tough fiber absorbs the $\sim 24.3 \mathrm{wt} \%$ of the water (Figure S8) and diameter increased 117\% (Figure S9). Figure S10 presented X-ray diffraction analysis was carried out for before and after contraction of the PU. A peak at around $2 \theta=20^{\circ}$ and a peak at $2 \theta=24^{\circ}$ is observed, which related to the crystallinity of

soft segments and hard segments, respectively. ${ }^{36}$ After contraction by the moisture, the hard segment relatively increases while soft segment decreased. It means that absorption of water molecules induces the reaggregation of the PU molecular chains. This contraction property could broaden wearable applications as being able to repair the tough fiber by impact or stretching force.

\section{Conclusion}

In conclusion, we have developed highly stretchable, and contractible tough fibers through the hybridization of GO, MWNT, and PU and prepared by a novel wet-spinning process. The bio-inspired tough fiber showed the highest toughness (494 J/g) using a 1:1 ratio of GO to MWNT and pre-stretched by $250 \%$. The toughness exceeds spider silk (165 J/g) and Kevlar (78 $\mathrm{J} / \mathrm{g}$ ). The reversible stretchability was demonstrated up to $80 \%$ strain and no significant change in deformation was observed after 100 stretching and releasing cycles. Furthermore, similar to spider silk, the tough fiber contracted $59 \%$ by exposure to high humidity and water. The maximum volumetric energy density during this contraction was $610 \mathrm{~kJ} / \mathrm{m}^{3}$ which is also higher than natural spider silk, reported moisture driven tensile actuators and the other actuators. 
Accordingly, this spider silk inspired stretchable and contractible tough fiber the broaden an application as a tough fiber for an energy absorption material, wearable devices, artificial muscle, and soft robotics.

\section{Experimental Section}

Materials. Graphene oxide (GO) dispersed aqueous solution with an average size of $37 \pm 23 \mu \mathrm{m}$ (monolayer with a thickness of ca. $0.81 \mathrm{~nm}$ ) was fabricated according to previously reported procedure. ${ }^{37,38}$ The multi-walled carbon nanotube $(<20 \mu \mathrm{m}$ length), and sodium dodecyl benzene sulfonate (SDBS) was purchased from Sigma-Aldrich (USA). Ether-based hydrophilic polyurethane (D640), which has $100 \%$ of linear expansion and ability of $90 \%$ water content, was purchased from AdvanSource biomaterials (USA).

Fabrication of the bio-inspired tough fiber. The stretchable and contractible tough fiber was fabricated as follows. First, a certain amount of GO and MWNT was dispersed in ethanol:DI water $(95: 5 \mathrm{v} / \mathrm{v})$ and $1 \mathrm{wt} \%$ of the SDBS was added. Then, GO/MWNT and SDBS mixture was sonicated for 1 hours to assist nano-particle dispersion and induce hydrogen bonds between GO and MWNT. After sonication, 8 wt $\%$ of PU added into the GO/MWNT dispersion and shaking by a vortex mixer continued overnight. The wet spinning to fabricate tough fibers used an extrusion speed of $4 \mathrm{ml} / \mathrm{hr}$ through a 26 -gauge needle from a syringe pump, and extruded bottom to top through a glass tube which contained DI water. The wet-spun gel-state fiber was dried at room temperature.

Characterization. The surface morphologies of the bio-inspired tough fiber were obtained by SEM (Hitachi S4700, Japan). Thermogravimetric analysis (TGA) (SDT Q600, TA instruments, USA) of the PU, GO/MWNT/PU fiber were measured from room temperature to $800{ }^{\circ} \mathrm{C}$ at rate of $10{ }^{\circ} \mathrm{C} / \mathrm{min}$ in $\mathrm{N}_{2}$ condition. The polarized Raman spectra were obtained using light polarized in the direction of fiber axis $\left(G_{\|}\right)$and compared to that for transverse direction polarization $\left(\mathrm{G}_{\perp}\right)$ with a Raman spectrometer (LabRam Aramis, Horiba Jobin Yvon, France) 
using $514.5 \mathrm{~nm}$ excitation. All stress-strain curves were measured by using a universal testing machine (Shimadzu model: EZ-SX, Japan), and tensile testing was performed a loading rate of $1 \mathrm{~mm} / \mathrm{min}$. The stress-strain curves of the wetted bio-inspired fiber were calculated by expanded diameter $(\sim 40 \mu \mathrm{m})$. Also, a training (stretching cycle) was applied before measuring the mechanical properties for increasing stability. X-ray diffraction patterns were measured by the diffraction angle $2 \theta$ from 20 to $60^{\circ}$ under a $\mathrm{Cu}$ radiation, voltage of $45 \mathrm{kV}$, and current of 200 mA using by X-ray diffractometer (Rigaku Model SmartLab, Japan). Moisture driven fiber contraction was operated by controlling the humidity in a closed environment and dried state fiber was carried out opened environment.

\section{Conflict of interest}

There are no conflicts to declare.

\section{Supporting Information}

Additional data for stress-strain curves, Raman spectra, SEM images, optical images, and actuation of the bio-inspired tough fiber. Movies demonstrating the fiber spinning, and contraction by humidity of the fiber.

\section{ORCID}

Seon Jeong Kim: 0000-0002-2867-6737

\section{Acknowledgements}

This work was supported by the Creative Research Initiative Center for Self-powered Actuation in National Research Foundation of Korea. Additional support was from the Australian Research Council and the Australian National Fabrication Facility. 


\section{References}

(1) Dalton, A. B.; Collins, S.; Muñoz, E.; Razal, J. M.; Ebron, V. H.; Ferraris, J. P.; Coleman, J. N.; Kim, B. G.; Baughman, R. H. Super-Tough Carbon-Nanotube Fibres. Nature 2003, 423, 703.

(2) Kim, H.; Kim, S. J. High Toughness of Bio-inspired Multistrand Coiled Carbon Nanotube Yarn. Carbon 2018, 131, 60-65.

(3) Miaudet, P.; Badaire, S.; Maugey, M.; Derré, A.; Pichot, V.; Launois, P.; Poulin, P.; Zakri, C. Hot-Drawing of Single and Multiwall Carbon Nanotube Fibers for High Toughness and Alignment. Nano Lett. 2005, 5, 2212-2215.

(4) Gonzalez, M. A.; Simon, J. R.; Ghoorchian, A.; Scholl, Z.; Lin, S.; Rubinstein, M.; Marszalek, P.; Chilkoti, A.; López, G. P.; Zhao, X. Strong, Tough, Stretchable, and SelfAdhesive Hydrogels from Intrinsically Unstructured Proteins. Adv. Mater. 2017, 29,1604743.

(5) Song, M.-M.; Wang, Y.-M.; Wang, B.; Liang, W.-Y.; Chang, Z.-Y.; Li, B.-J.; Zhang, S. Super Tough, Ultrastretchable Hydrogel with Multistimuli Responsiveness. ACS Appl. Mater. Interfaces 2018, 10, 15021-15029.

(6) Javadi, M.; Gu, Q.; Naficy, S.; Farajikhah, S.; Crook, J. M.; Wallace, G. G.; Beirne, S.; Moulton, S. E. Conductive Tough Hydrogel for Bioapplications. Macromol. Biosci. 2018, 18, 1700270.

(7) Yuk, H.; Zhang, T.; Lin, S.; Parada, G. A.; Zhao, X. Tough Bonding of Hydrogels to Diverse Non-Porous Surfaces. Nat. Mater. 2016, 15, 190-196.

(8) Morelle, X. P.; Illeperuma, W. R.; Tian, K.; Bai, R.; Suo, Z.; Vlassak, J. J. Highly Stretchable and Tough Hydrogels below Water Freezing Temperature. Adv. Mater. 2018, 30, 1801541.

(9) Chen, K.; Shi, B.; Yue, Y.; Qi, J.; Guo, L. Binary Synergy Strengthening and Toughening of Bio-Inspired Nacre-like Graphene Oxide/Sodium Alginate Composite Paper. ACS Nano 2015, 9, 8165-8175. 
(10) Zhang, Y.; Li, Y.; Ming, P.; Zhang, Q.; Liu, T.; Jiang, L.; Cheng, Q. Ultrastrong Bioinspired Graphene-Based Fibers via Synergistic Toughening. Adv. Mater. 2016, 28, 28342839.

(11) Shin, M. K.; Lee, B.; Kim, S. H.; Lee, J. A.; Spinks, G. M.; Gambhir, S.; Wallace, G. G.; Kozlov, M. E.; Baughman, R. H.; Kim, S. J. Synergistic Toughening of Composite Fibres by Self-Alignment of Reduced Graphene Oxide and Carbon Nanotubes. Nat. Commun. 2012, 3, 650.

(12) Vollrath, F.; Knight, D. P. Liquid Crystalline Spinning of Spider Silk. Nature 2001, 410, 541-548.

(13) Boutry, C.; Blackledge T. A. Evolution of Supercontraction in Spider Silk: StructureFunction Relationship from Tarantulas to Orb-Weavers. J. Exp. Biol. 2010, 213, 3505-3514.

(14) Wu, Y.; Shah, D.U.; Wang, B.; Liu, J.; Ren, X.; Ramage, M. H.; Scherman, O. A. Biomimetic Supramolecular Fibers Exhibit Water-Induced Supercontraction. Adv. Mater. 2018, 30, 1707169.

(15) Liu, Y.; Shao, Z.; Vollrath, F. Relationships Between Supercontraction and Mechanical Properties of Spider Silk. Nat. Mater. 2005, 4, 901-905.

(16) Kim, S. H.; Kwon, C. H.; Park, K.; Mun, T. J.; Lepró, X.; Baughman, R. H.; Spinks, G. M.; Kim, S. J. Bio-Inspired, Moisture-Powered Hybrid Carbon Nanotube Yarn Muscles. Sci. Rep. 2016, 6, 23016.

(17) Ma, M.; Guo, L.; Anderson, D. G.; Langer, R. Bio-Inspired Polymer Composite Actuator and Generator Driven by Water Gradients. Science 2013, 339, 186-189.

(18) Agnarsson, I.; Dhinojwala, A.; Sahni, V.; Blackledge, T. A. Spider Silk as a Novel High Performance Biomimetic Muscle Driven by Humidity. J. Exp. Biol. 2009, 212, 1990-1994. 
(19) Madden, J. D. W.; Member IEEE,; Vandesteeg, N. A.; Anquetil, P. A.; Madden, P. G. A.; Takshi, A.; Pytel, R. Z.; Lafontaine, S. R.; Wieringa, P. A.; Hunter, I. W. Artificial Muscle Technology: Physical Principles and Naval Prospects. IEEE J. Oceanic Eng. 2004, 29, 706-728. (20) Wang, J.; Hu, J.; Wen, Y.; Song, Y.; Jiang, L. Hydrogen-Bonding-Driven Wettability Change of Colloidal Crystal Films: From Superhydrophobicity to Superhydrophilicity. Chem. Mater. 2006, 18, 4984-4986.

(21) Breuer, O.; Sundararaj, U. Big Returns from Small Fibers: A Review of Polymer/Carbon Nanotube Composites. Polymer Composites, 2004, 25, 630-645.

(22) Liu, Y.; Sponner, A.; Porter, D.; Vollrath, F. Proline and Processing of Spider Silks. Biomacromolecules 2008, 9, 116-121.

(23) Han, J.-H.; Zhang, Hui.; Chen, M.-J.; Wang, G.-R.; Zhang, Z. CNT Buckypaper/Thermoplastic Polyurethane Composites with Enhanced Stiffness, Strength and Toughness. Compos. Sci. Technol. 2014, 103, 63-71.

(24) Coleman, J. N.; Cadek, M.; Blake, R.; Nicolosi, V.; Ryan, K. P.; Belton, C.; Fonseca, A.; Nagy, J. B.; Gun'ko, Y. K.; Blau, W. J. High-Performance Nanotube-Reinforced Plastics: Understanding the Mechanism of Strength Increase. Adv. Funct. Mater. 2004, 14, 791-798.

(25) Cheng, M.; Chen, W. Mechanical Properties of Kevlar ${ }^{\circledR}$ KM2 Single Fiber. J. Eng. Mater. Technol. 2005, 127, 197-203.

(26) Hu, D.; Gong, W.; Di, J.; Li, D.; Li, R.; Lu, W.; Gu, B.; Sun, B.; Li, Q. Strong GrapheneInterlayered Carbon Nanotube Films with High Thermal Conductivity. Carbon 2017, 118, 659665.

(27) Wang, R.; Sun, J.; Gao, L.; Xu, C.; Zhang, J. Fibrous Nanocomposites of Carbon Nanotubes and Graphene-Oxide with Synergetic Mechanical and Actuative Performance. Chem. Commun. 2011, 47, 8650-8652. 
(28) Seyedin, M. Z.; Razal, J. M.; Innis, P. C.; Jalili, R.; Wallace, G. G. Achieving Outstanding Mechanical Performance in Reinforced Elastomeric Composite Fibers Using Large Sheets of Graphene Oxide. Adv. Funct. Mater. 2015, 25, 94-104.

(29) Nah, C.; Lim, J. Y.; Cho, B. H.; Hong, C. K.; Gent, A. N. Reinforcing Rubber with Carbon Nanotubes. J. Appl. Polym. Sci. 2010, 118, 1574-1581.

(30) Faber, K. T.; Evans, A. G. CRACK DEFLECTION PROCESSES-I. THEORY. Acta. Metall. 1983, 31, 565-576.

(31) Rafiee, M. A.; Rafiee, J.; Srivastava, I.; Wang, Z.; Song, H.; Yu, Z.-Z.; Koratkar, N. Fracture and Fatigue in Graphene Nanocomposites. Small 2010, 6, 179-183.

(32) Jin, L.; Bower, C.; Zhou, O. Alignment of Carbon Nanotubes in a Polymer Matrix by Mechanical Stretching. Appl. Phys. Lett. 1998, 73, 1197-1199.

(33) Gommans, H. H.; Alldredge, J. W.; Tashiro, H.; Park, J.; Magnuson, J.; Rinzler, A. G. Fiber of Aligned Single-Walled Carbon Nanotubes: Polarized Raman Spectroscopy. J. Appl. Phys. 2000, 88, 2509-2514.

(34) Mullins, L. Effect of Stretching on the Properties of Rubber. Rubber Chem. Technol. 1948, 21, 281-300.

(35) Aliev, A. E.; Oh, J.; Kozlov, M. E.; Kuznetsov, A. A.; Fang, S.; Fonseca, A. F.; Ovalle, R.; Lima, M. D.; Haque, M. H.; Gartstein, Y. N.; Zhang, M.; Zakhidov, A. A.; Baughman, R. H. Giant-Stroke, Superelastic Carbon Nanotube Aerogel Muscles. Science, 2009, 323, 1575 1578.

(36) Khosravi, A.; Sadeghi, M.; Banadkohi, H.; Talakesh, M. M. Polyurethane-Silica Nanocomposite Membranes for Separation of Propane/Methane and Ethane/Methane. Ind. Eng. Chem. Res. 2014, 53, 2011-2021.

(37) Jalili, R.; Aboutalebi, S. H.; Esrafilzadeh, D.; Shepherd, R. L.; Chen, J.; Yamini, S. A.; Konstantinov, K.; Minett, A. I.; Razal, J. M.; Wallace, G. G. Scalable One-Step Wet-Spinning 
of Graphene Fibers and Yarns from Liquid Crystalline Dispersions of Graphene Oxide: Towards Multifunctional Textiles. Adv. Funct. Mater. 2013, 23, 5345-5354.

(38) Jalili, R.; Aboutalebi, S. H.; Esrafilzadeh, D.; Konstantinov, K.; Razal, J. M.; Moulton, S. E.; Wallace, G. G. Formation and Processability of Liquid Crystalline Dispersions of Graphene Oxide. Mater. Horiz. 2014, 1, 87-91. 


\section{Figures}

a)

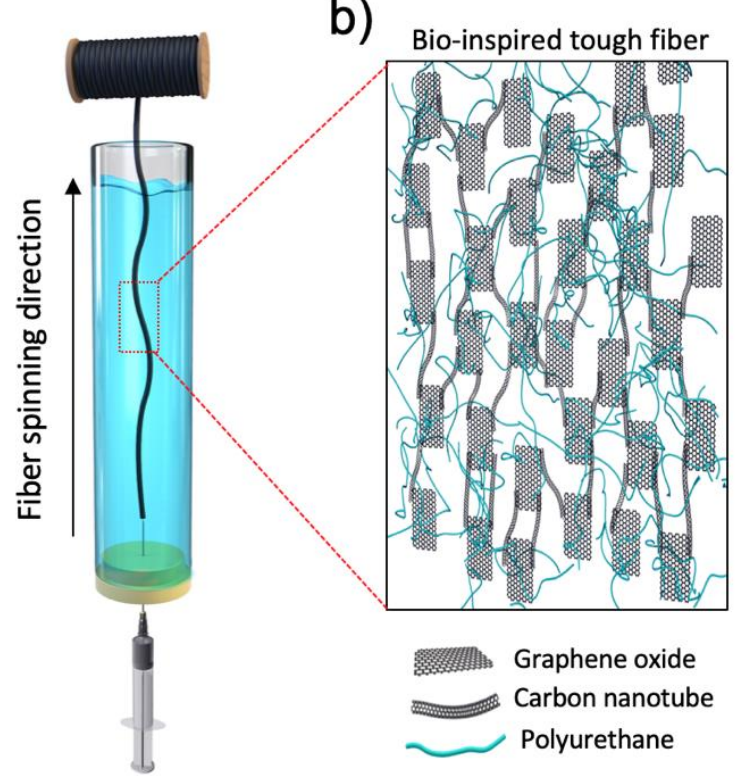

c)

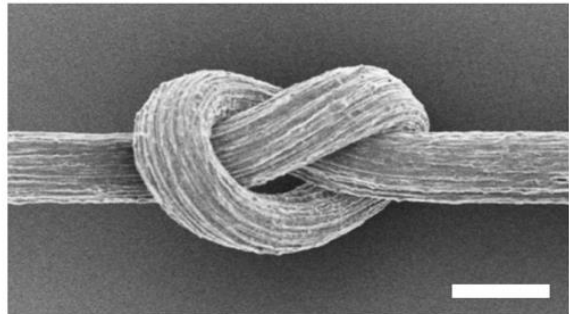

d)

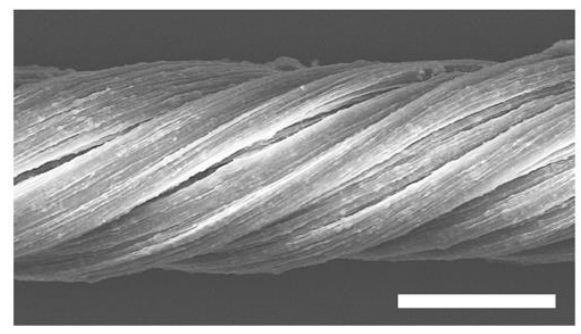

Figure 1. Schematic images of a) the wet spinning method in which the bio-inspired tough fiber floats while coagulating from the bottom to top in the glass tube filled with DI water. b) Illustration of the structure of the tough fiber composed of GO/MWNT and PU. SEM images of c) the knotted tough fiber (scale bar: $25 \mu \mathrm{m}$ ), and d) 10-plied tough fiber twisted to 5000 turns $/ \mathrm{m}$. (scale bar: $50 \mu \mathrm{m}$ ). 

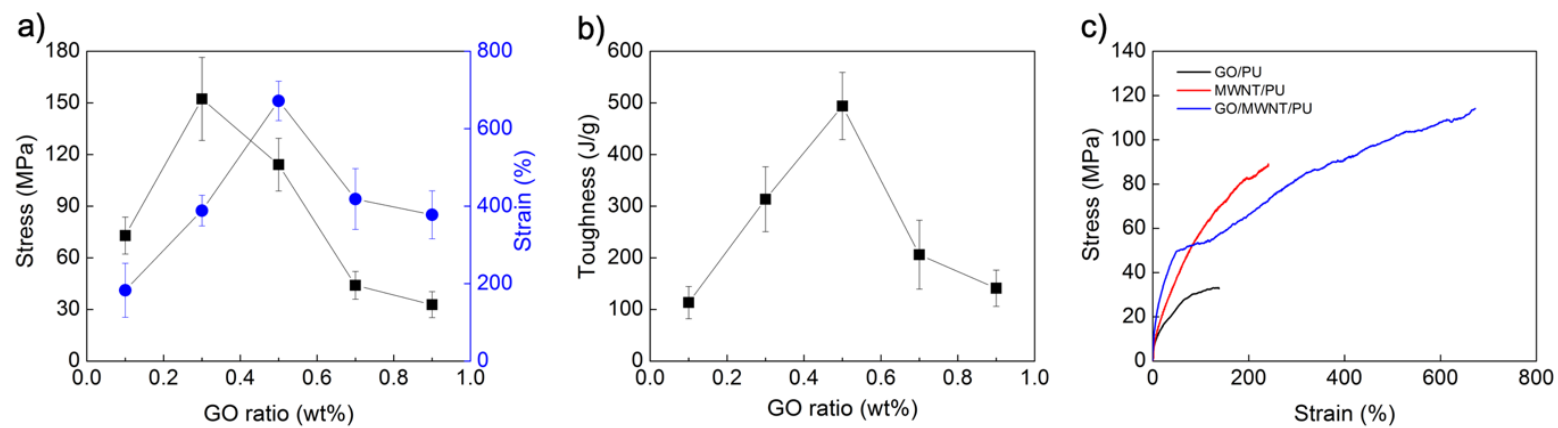

Figure 2. Mechanical properties of the dried tough fibers. a) Maximum tensile stress and strain at break, and b) toughness value of the tough fiber with different weight ratios of GO/MWNT. The combined weight fraction of nano-carbons (GO and MWNT) in the tough fibers was maintained at $12.5 \%$ for all fibers. c) Stress-strain curves of the GO/PU, MWNT/PU, and tough fiber. All mechanical properties were measured with a loading rate of $1 \mathrm{~mm} / \mathrm{min}$ and fiber length is $10 \mathrm{~mm}$. 
a)

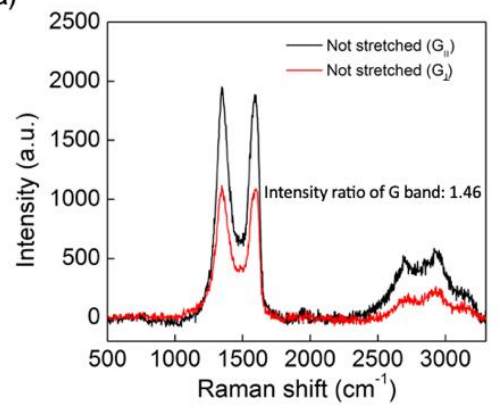

b)

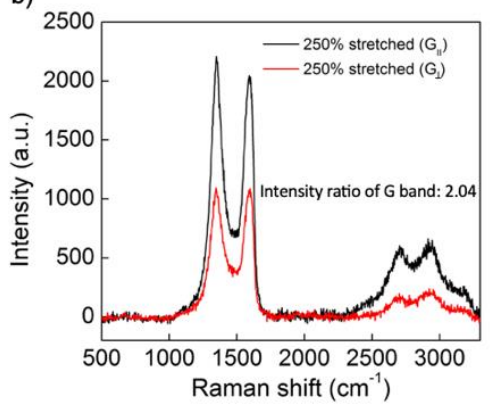

c)

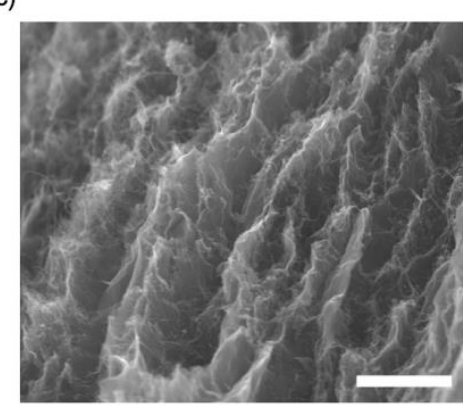

Figure 3. Polarized Raman spectra for the intensity ratio of the $\mathrm{G}$ band of a) not stretched and b) $250 \%$ stretched tough fiber for light polarized in the direction of the tough fiber axis $\left(\mathrm{G}_{\|}\right)$ and transverse direction polarization $\left(\mathrm{G}_{\perp}\right)$. c) Cross-sectional SEM image of the tough fiber without PU. (Scale bar: $2 \mu \mathrm{m}$ ) 

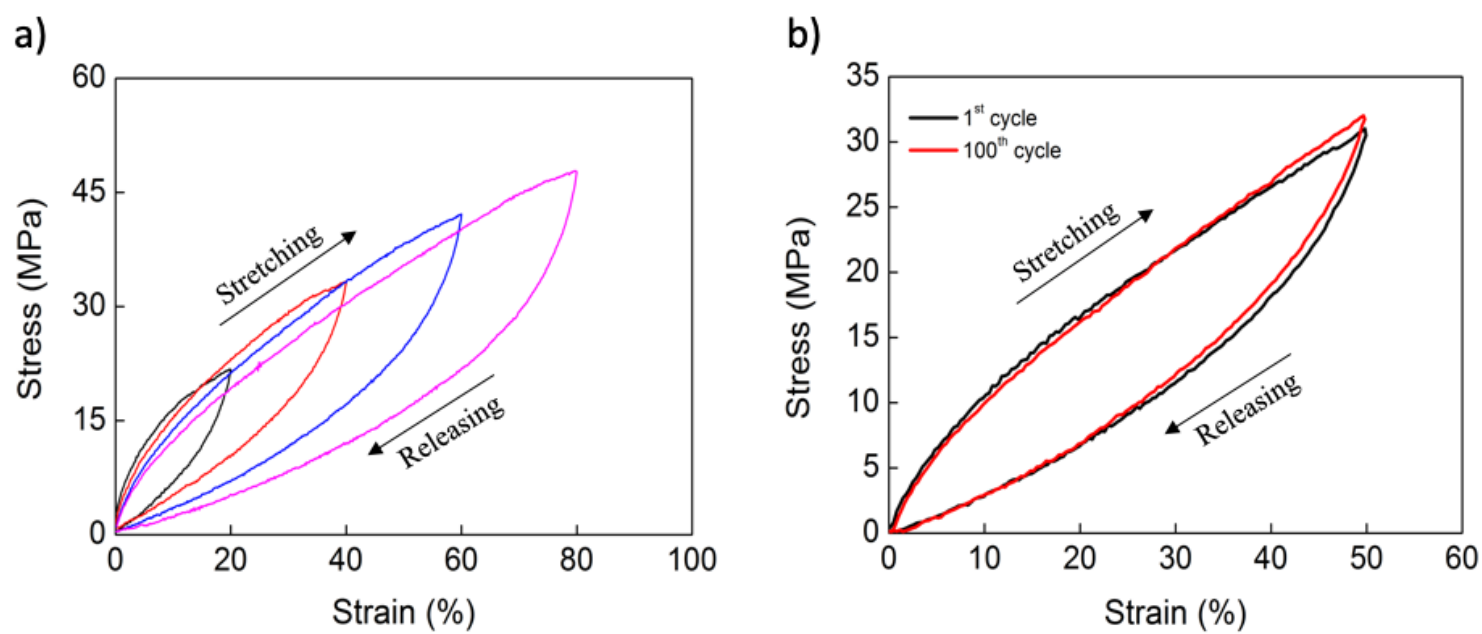

Figure 4. Stress-strain curves of stretching and releasing for the tough fiber. a) The fiber was stretched and released to increasing maximum strains of 20, 40,60, and $80 \%$. b) Comparison of the $1^{\text {st }}$ and the $100^{\text {th }}$ repeated stretch and relax cycle to a maximum strain of $50 \%$. 


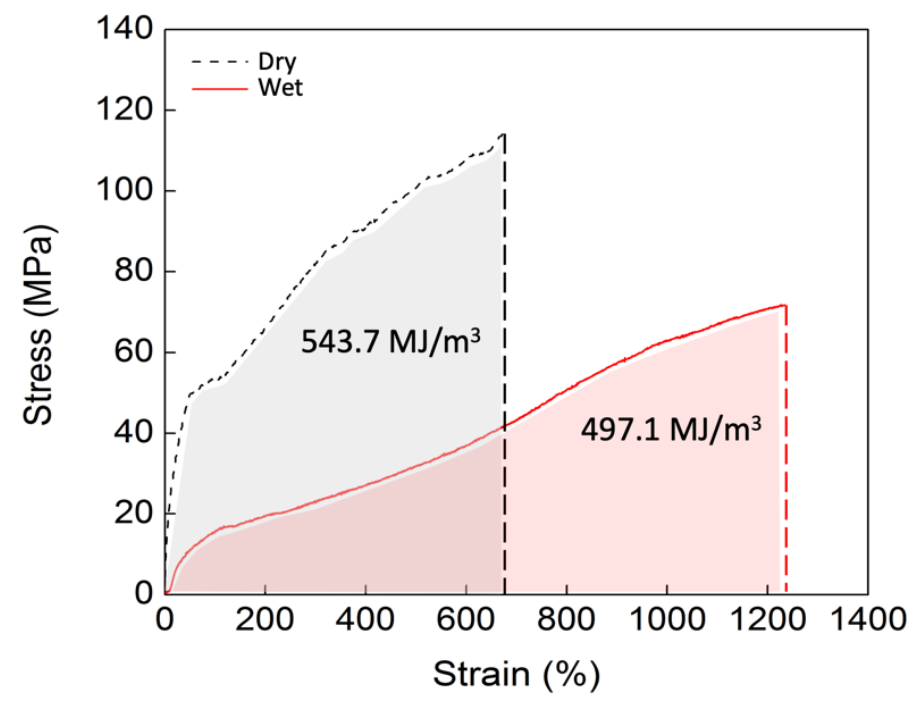

Figure 5. Comparison of stress-strain curves of the dried and wet state contracted tough fiber. The area represents volumetric toughness. 
a)

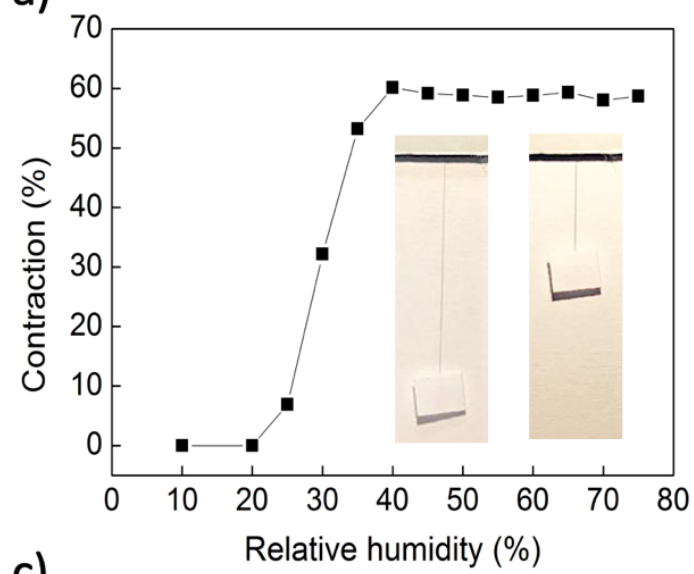

c)

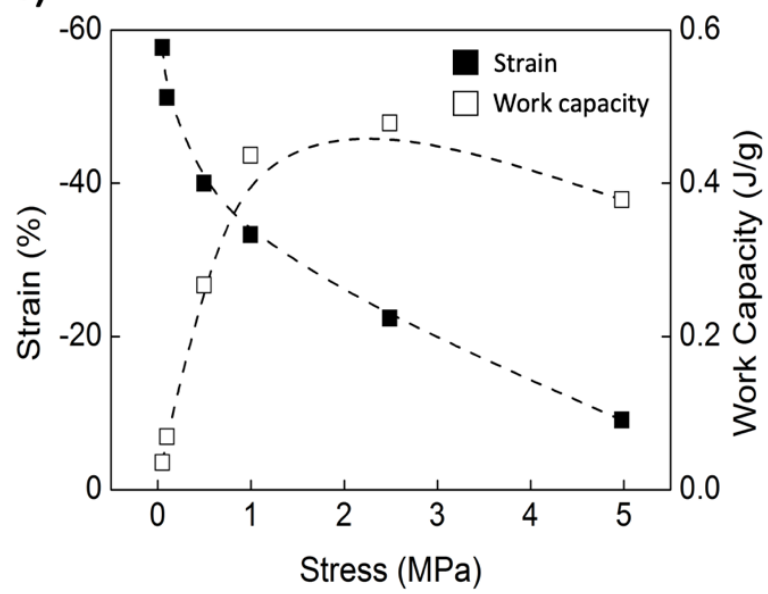

b)

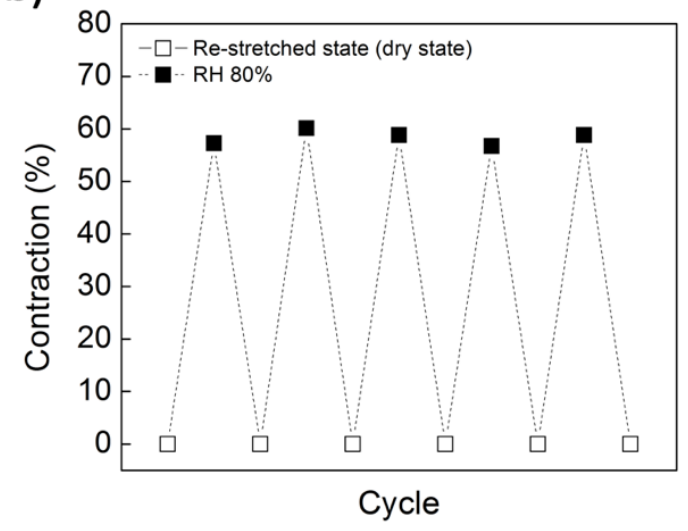

d)

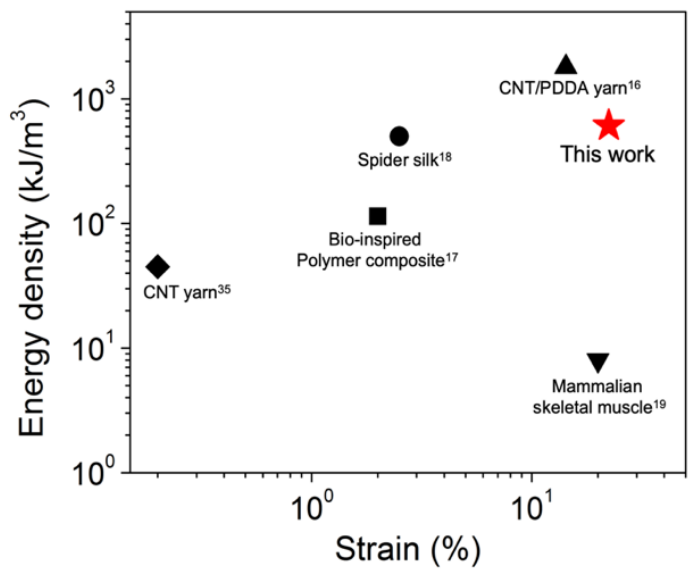

Figure 6. a) The contraction of the tough fiber versus relative humidity. The inset shows the optical images of the tough fiber before and after contraction by the moisture under $0.1 \mathrm{MPa}$ load. b) Repeatability of the moisture induced contraction. Mechanical stress was applied to the tough fiber after contraction and drying to return to the initial length. c) The stress dependence of tensile contraction and work capacity driven by the water. d) Comparison of the energy density of the actuator and stroke for the bio-inspired tough fiber. ${ }^{16-19,35}$ 
Graphic for manuscript (Table of Contents)
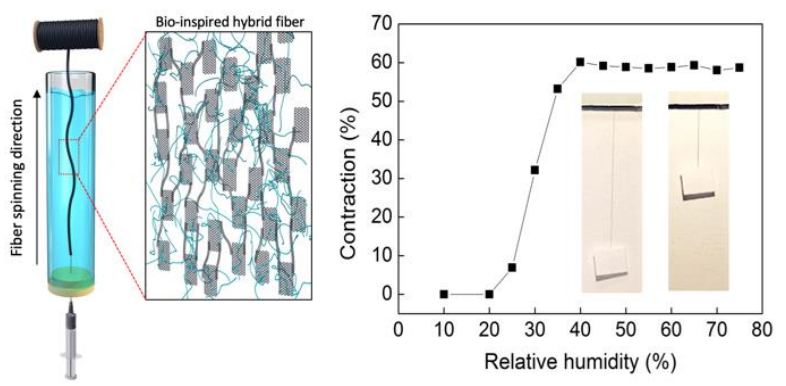